\title{
AUC-SAS: Integrated analysis of biomacromolecular structure with analytical ultracentrifugation (AUC) and small-angle scattering (SAS)
}

\author{
K. Morishima, R. Inoue, M. Shimizu, A. Okuda, N. Sato, R. Urade, M. Sugiyama \\ Institute for Integrated Radiation and Nuclear Science, Kyoto University, 2-1010 Asashironishi, \\ Kumatori, Sennan, Osaka, 590-0494, Japan \\ morishima@rri.kyoto-u.ac.jp
}

Small-angle X-ray and neutron scatterings (SAXS and SANS; collectively called SAS) offer overwhelming opportunities for structural analysis of a biomacromolecule in solution. Modern SAS analysis with a computational simulation provides a three-dimensional structural model, whereas it is essential for the high-quality analysis to obtain the scattering profile surely corresponding to a target molecule. However, an undesirable aggregate, even at the low weight fraction, deteriorates the scattering profile of the target molecule and then lead to failure of the structural analysis. To overcome the aggregation-problem, we have developed the integrated approach with analytical ultracentrifugation (AUC) and SAS, namely "AUC-SAS"[1].

Figure 1 demonstrates the aggregation-removal with AUC-SAS for a bovin serum albumin (BSA) solution including the aggregates. AUC revealed the weight fractions of the monomer and their aggregates in the solution (Figure 1a). Because SAS offered the scattering profile ensemble-averaged over the monomer and their aggregates (open circles in Figure 1b), the simple experimental scattering profile led to the incorrect structural model as the monomer. AUC-SAS derived the scattering profile of the monomer (closed circles in Figure 1b) from the simple experimental one utilizing the information of AUC. Consequently, the derived profile led to the reasonable structural model as the monomer. In the recent progress, AUC-SAS succeeded for the solution including aggregates up to $20 \%$ of weight fraction.

AUC-SAS does not require a large amount of sample nor very high intensity beam compared with size exclusion chromatographySAXS (SEC-SAXS). Therefore, AUC-SAS has a potential as a complementary method for laboratory-based SAXS and standard SANS. The software for AUC-SAS data reduction is available at http://www.rri.kyoto-u.ac.jp/NSBNG/activity.html.
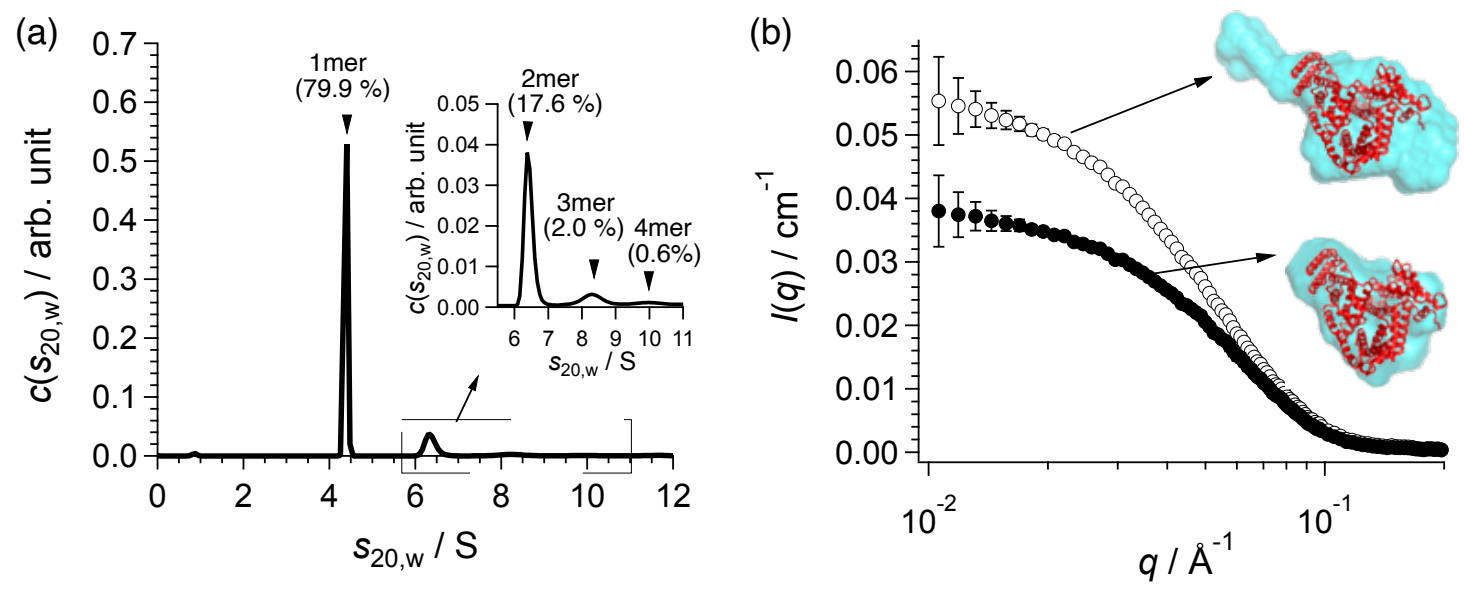

Figure 1. Demonstration of AUC-SAS for bovin serum albumin (BSA) solution: (a) Weight fraction distribution in the BSA solution obtained from AUC. (b) Open and closed circles indicate the SAXS profile for the BSA solution and AUC-SAS-treated profile, respectively. Inset shows the three-dimensional structural models calculated from the scattering profiles with DAMMIN[2] (cyan) and the crystal structure of BSA monomer (red).

[1] Morishima, K., Okuda, A., Inoue, R., Sato, N., Miyamoto, Y., Urade, R., Yagi-Utsumi, M., Kato, K., Hirano, R., Kujirai, T., Kurumizaka, H. \& Sugiyama, M. (2020). Communications Biology 3, 294.

[2] Svergun, D. I. (1999). Biophysical journal 76, 2879.

Keywords: Analytical ultracentrifugation (AUC); Small-angle scattering (SAS); AUC-SAS; Aggregation; Biomacromolecule 